\title{
Semantics of Portions and Partitive Nouns for NLP
}

\author{
Salvador Climent \\ Facultat de Filologia, Secció Lingüística General \\ Universitat de Barcelona \\ Gran Via 585 \\ 08007 Barcelona \\ Spain \\ climent $(\Theta)$ goliat.upc.es
}

\begin{abstract}
This paper describes a system of representation of nouns denoting portions, segments and relative quantities of entities, in order to account for this case of part-whole relationship. The semantics of both constructions denoting portions and nouns used to build them are discussed and eventually formalised in a unification-based formalism (I,KB-I,RL) in terms of Pustejovsky's Theory of Qualia and Iackendof's Conceptual Scmantics.
\end{abstract}

\section{Introduction and Motivation}

Processes of inference of meaning concerning partwhole relations can be drawn from a lexicon bearing meronymic links between words (cl. [W $\Lambda \mathrm{I} .88]$ ) e.g. component-whole links (hand-arm), membercollection (tree-forest) and so. The case of portions, segments and relative quantities of objects or substances (slices, lumps, buckets, spoonfuls, grains, tops or tips of things) is the exception, since this is a relation which is encoded in the language by means of constructions. Contrary to that which some authors posit ([CIIA88], [WIN87]), it doesn't seem to be a productive linguistic gencralisation to set in a lexicon some part-of link between slice and cake. In any case, such relation would exist between cake and slice of cake -namely, the part-of relation should stand between slice and any slicerble thing.

Generally speaking, the relation portion may be characterised, semantically, in that the whole preexists the part and the part retains most of the properties of the whole ([IRI88]), and, syntactically, as surfacing in $\langle\mathrm{X}$ of $\mathrm{NP}\rangle$ structures, being potentially $\langle\mathrm{X}\rangle$ an apparently heterogeneous collection of words which, henceforth, following [COI ${ }^{\mathrm{S}} 2$ 2] We will refer to as l'artitive Nouns (PNs).

On the other hand, portions denoted by such constructions differ from their wholes in some aspects, basically individuation, quantity, process of bringing about, and shape. Such properties, since they are present in $\langle\mathrm{X}$ of $\mathrm{NI}\rangle$ but they were not in $\langle\mathrm{NP}\rangle$, are assumed to be carricd to the construction by the noun $(<\mathrm{X}>)$.

I'll try to show here that it is plausible to give a global account of such heterogeneous set of words, since they bear a range of common and distinctive linguistic features, and I'll try to provide a representation feasible for NLP which account both for PNs as a general class and for the homogeneous subclasses which within them could be distinguished and delined 1

\section{Framework}

We assume some general familiarity with the framework We work within: LKB-I,RI. ([COP')2]) as a formalism of computational representation, The (iencrative Lexicon ([PYSS95]) as a theoretical ground for the formalisation of lexical signs, and Conceptual Semantics ([JAC91]) for the conceptualisation of parts and boundaries of entities.

LKB-IRL is an IIPSG-like formalism based on typed feature structures ([C.AR92]) developed within the Acquilex Project which makes use of unification and mechanisms of default inheritance, There are four basic levels of representation: OR'TH, C.AT', SEM and RQS. CAT encodes syntax using a categorial grammar approach, so there are simple (c.g. noun: $\mathrm{N}$ ) and complex (c.g. adjective: N/N) categorics. It is to be noticed that in complex categories the active element is not a category but a lexical sign, in a way that selective restrictions may be set at this level by specifying the semantic type or other features of the selected sign. SEM encodes logical semantics. RQS is basically equivalent to Pustejovsky's Qualia Structure (henecforth QUALIA).

The Theory of Qualia is embedded in that of the Gencrative Lexicon and has as a basic aim to improve compositionality by endowing lexical signs with a rich internal structure which accounts for different facets of human knowledge about lexical-semantic content, in a way that, in many cases, it allows for avoiding listing separate cntries to account for lexical polysemy. [PUS95] posits the lexical entry to be constituted by four structures: Event, Argument, Icxical-Inheritance and QUALIA. The latter consists of four Quales: Agentive (origin or 'bringing alsout' of objects), Telic (purpose and function), Formal (that which distinguishes it within a larger domain: magnitude, shape, dimensionality) and Constitutive (relation between an object and its constituents or

I This work has been developped for Spanish. Notwithstanding, for case of exposition, I'll exemplify the discussion by means of examples of English - when possible. In any case, for what concerns us here, Spanish and English are, both semantically and structurally, strongly comparable the kemel $\langle\mathrm{PN}$ of $\mathrm{NP}\rangle$ comes out in Spanish as a literal translation, $\langle\mathrm{IN}$ de NP$\rangle$. 
proper parts).

[IAC91] posits that speakers understand and schematise a range of phenomena such as mass-count distinction, plurality, partitive constructions and boundary words in terms of elementary conceptual features such as dimensionality, idealised boundaries and internal structure. He introduces features $[ \pm B]$ (boundaries being or not in view or of concern) and [ \pm I] (entailment or not about internal structure) for both objects and events as expressing the gencrality of phenomena of boundedness and plurality the notion of $\pm \mathrm{I}$ is present in one or another way in [T/L 78] (cf. [IAC91]), [KRI87] (cf. [COP92]) and [LAK87]; that of boundedness in [I AN91]. This feature system comes out as:

$+\mathrm{B}-\mathrm{I}$ : individuals (a pig)/closed events (John ran to the store)

$+\mathrm{B}+\mathrm{I}$ : groups (a committee)/bounded ilcrative events (the light flashed until down)

-B-I: substances (water)/ unbounded homogencous processes (. \%ohn slept)

- $\mathrm{B}+\mathrm{I}$ : aggregates (buses, cattle)/ unbounded iterative processes (the light flashed continually)

Moreover, he posits that boundaries of entities are conceptualised as minimal claborations (notated $e$ ) of their ideal topological boundaries (i.c.the tip of the tongue is the portion which corresponds to a minimal elaboration of the 0 -dimensional point which would ideally be the tonguc's tip).

\section{Portions and Partitive Nouns}

$A$ portion designs a bounded region within a domain ([LAN91]), hence the portion is an individuated entity (even in the case the whole be a substance or mass). The syntactic effect is that, as pointed out in [VOS94], the construction which denotes the portion is syntactically countable.

$\Lambda$ portion, an individuated (bounded), object has a shape different from that of the whole. This information is contributed to the construction by the PN. There are PNs which clearly specily shape (RODAIA, lump) while others underspecify it (fragment). In many cases, PNs, as adjectives do, predicate properties of the portion, specially shape (the Iranslation of Spanish RODAIA must be the paraphrase round slice; a lath of anything is saliently elongated) or size, but also thickness, consistency or others (as in the case of MENDRUGO, equivalent 10 portion (of bread) except for the fact that entails that the bread is not fresh but stale).

$\Lambda$ portion always conveys a measure with relation to the total magnitude of the whole. 'Therefore, nouns such as bucket, slice, lump or grain are relative quantifiers in the sense of [I.AN91]:

a relative quantifier is so-called because it specifies a quantity in relation to a reference. mass; in the default-case interpretation, this reference mass consists of the maximal instantiation of the pertinent category (i.e. its full extension in all conceivable worlds).

A portion has been oblained by a different process than the whole: a cake has been obtained by baking it, but a slice of cake by cutting it of the cake. The meaning of portions is many times tightly related to such agentive process -if one has obtained 'slices' it necessarily has been by cutting something; there even exists the verb to slice. As pointed out in [PUS95], knowledge of 'coming into being' is a property of nominals which has remarkable linguistic consequences sinec it can account for a reduction of lexical polysemy. For instance, the Spanish phrase HACJ:R PAN [to-make bread] means baking bread, whilst HACIR RIBBANADAS (DE: PAN) [to-make slices (of bread)] means slicing (bread). This way, the very same verb HACER shows two radically different meanings, which in principle should be listed separately in the lexicon. Nevertheless, both can be accounted for in a single entry which selects the Agentive Role of the complement.

More specifically talking about the lexical signs we call PNs, [LYO77] shows that they correspond to the classifiers in languages as Tzcltal, Mandarin Chinese or Vietnamese. In languages wilh classificrs, these words, semantically strongly similar to determiners and quantifiers, have functions of individuation and enumeration, making surface notions such as sort of entity, shape or measure. E.g. the Chinese phrase SAN BEN SIIU is translatable by three plane-entity book; three whiskies would be constructed with a mensural classifier, being the translation paraphrasable by three unit/doses whisky. [I. YO77] makes notice that words such as head (of cattle), sheet (of paper) or lump (of sugar) stand for exactly the same function as classificrs in those languages. Specilically, lu $\mathrm{mp}$ operates simulancously as both a mensural (meaning conventional dose) and classal (denoting a certain type of aggregale) classificr.

Some have assumed that PNs select mass nouns (slice of cake, glass of wine), being mass nouns the way in which substances tipically surface in the language. Instead, we posit that PNs select both kinds of nouns (count or mass) denoting both kinds of things (individuals or substances), but in any case, crucially, surfacing as expressing cumulative reference

Let's consider RODAJA DE I.IMON [round-slice of lemon]. To assume that IIMON here is mass cntails assuming that it has undergone a derivational 'grinding' rule which converts countable individuals in masses. Nevertheless, a round-slice of lemon is always a slice of some individual lemon, not a special measure of substance which some time in the past was 'lemon'. In any case, if a 'lemon' weren't an individuated and bounded thing, it couldn't be sliced and the shape of the portion wouldn't depend on that of the whole. The confusing point is that IJIMON in the example, ROD $\triangle J A$ DI: I.IMON, surfaces grammatically as substances usually do -namely, zerodetermined. But zero-determination is not exclusively a ressource to refer to substances, it is the way of expressing cumulative reference. Both individuals and substances may be refered to cumulatively, that is, be construed as an indifierentiated amassment. This surfaces in the language as a zero-determiner plus the 
noun in singular in the case of substances (a glass of wine), and either in singular or plural in the case of individuals (a slice of lemon, a basket of lemons).

So, in our point of view PNs tipically select the noun of the whole ats it surlaces when construing cumulative reference -but this doesn't compulsorily entails neither the referent is a substance nor it is refered to by means of a mass noun. In the case of individuals, referents still are bounded things, hence both they can be sliced and the shape of their portions still can depend on that of the original whole. We can't go further with the issue here but, at last, what the discussion above stands for is that human conceptualisation is considered as the cause, and the mass-count distinction, as the surface effect.

PNs are not straightforwardly referential, as they predicate with reference to another entity. 'This may be noticed considering sentences such as ?? Jolln ate a slice or ??I dranght three cups. They are scmantically uncomplete as they don't allow the hearer to retrieve from them the information the speaker wants to convey. liurther information as in John ate a slice of cake or It was an excellent coffee. I draught three cups is needed to do the lask. When appearing in the discourse, PNs need of further specification of the referent, either via of-complementation or via ellipsis or anaphora. Consecpuently, they can not be unary but relational predicates in the sense of $[I . \Lambda N 91]$, that is, terms which are predicales only with reference 10 some other entity.

$\Lambda$ basic linguistic feature of $\mathrm{PNs}$ is that they, as relational predicates, bear selectional restrictions. Namely, cach kind of PN can combine with certain referential nouns but can not combine with others, depending on certain features of the referential noun. These features are mostly linguistic (type, countability, singular or plural) but also can depend on knowledge of the world (physical state, etc.). We hypothesise that, in general, distinctions between classes of PNs concerning selectional restrictions must be due to linguistic reasons, while further specilications within each class would be duc (1) properties of the referent. li.g., it could be assumed that containers (cups, baskets) select $[-\mathrm{B} \mid$ items (substances and plurals), and more specifically, cups select liquids and baskets non-licuids.

\section{Sorts of PNs}

We are not committed here to represent individuation of groups (team, committee) or aggregates (cattle, furniture). In our background ontology, which is intended to represent things in the world as conceptualised by humans (fig.1), these constitute a different class since, in this class of words, speakers' conceptualisation focuses not on the element entities but in their aggregation. Plunals ane considered as representing an aggregation (Iherefore a $-\mathrm{B}+\mathrm{I}$ concept) of bounded things (therefore either individuals or groups) by means of a derivative (lexical) rule applice on signs denoting those elements (fig.2) - c.g. cow $\rightarrow>$ cows, team $->$ teams; but gold,cattle $->$ *golds/*attles).

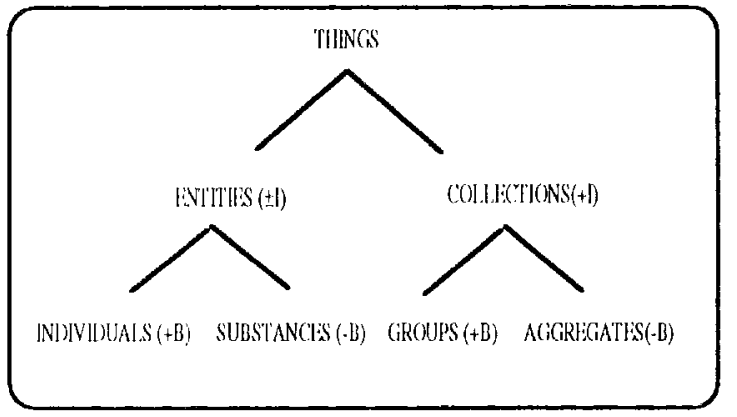

Figure 1. Ontology

Intity-portioning terms have been sorted as follows:

Contents (henceforth CONT) (a bucket of water, a basket of wheat, a basket of lemons). They are metonymies of containers expressing a conventionalised measure or quantity of a $[-B]$ entily. Shape is not relevant -a bathtub may contain a bucket of water without there being any bucket in it $([\mathrm{I}, \Lambda \mathrm{N}) 1])$

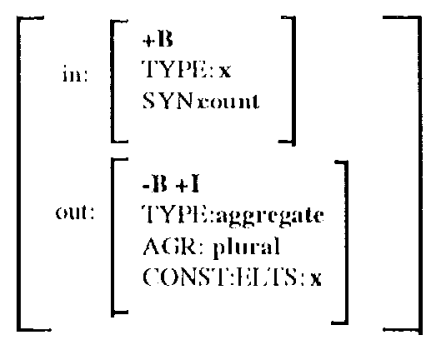

ligure 2. Pluralization (Lexical) Rule wements (LIT) (UN (iAJ) DI: IIMON lan 'inner-preexistent-division-ol-some-fruits'of lemon], a grain of rice). They are individuations of pre-existing parts of the whole. They select $[+1]$ entities, either individuals or substances. They are not committed to an $\Lambda$ gentive process as they may remain attached to the whole. Shape and measure, if considered relevant, are inherent to the portion itself

Boundaries (BOUNI) (the tip of the tongue, the surface of the sea, the top of $a$ box). They are idealisations of physical boundaries of the whole. Select $[+B]$ entities. They are non- $\Lambda$ gentive either. Their shape is tightly related to that of the whole but one of their dimensions is conceptualised as close to non-existence ([I^C9[]). Analogously, they denote a minimal quantity of the whole.

Both Detached (D'TCII) (a slice of cake, a slice of lemon) and Modelled (MDL.D) portions (a lump of sugar, a sheet of perper) have been drawn out of the whole and bear a shape straighlforwardly determined by such $\Lambda$ gentive process. The meaning of the former focus on the $\Lambda$ gentive, which is a process of cut or fragmentation of a $[+B$ solid. The latter focus on shape, which is often conceptualised schematically(sheet: a plane; lump, ingot: brickshaped). The $\Lambda$ gentive is a proeess of modelling of a $[-B]$ substance.

Summarising about seleclional restrictions of these sorts of PNs, they come out as follows:

EI.T: select $[+I]$ entities (=individuated or substance) (a 'pre-cxisting-inner-division' of IIMON, wheat, sugar)

BOUND: $[+B]$ entilics (=individuated) (a 'boundary'of 


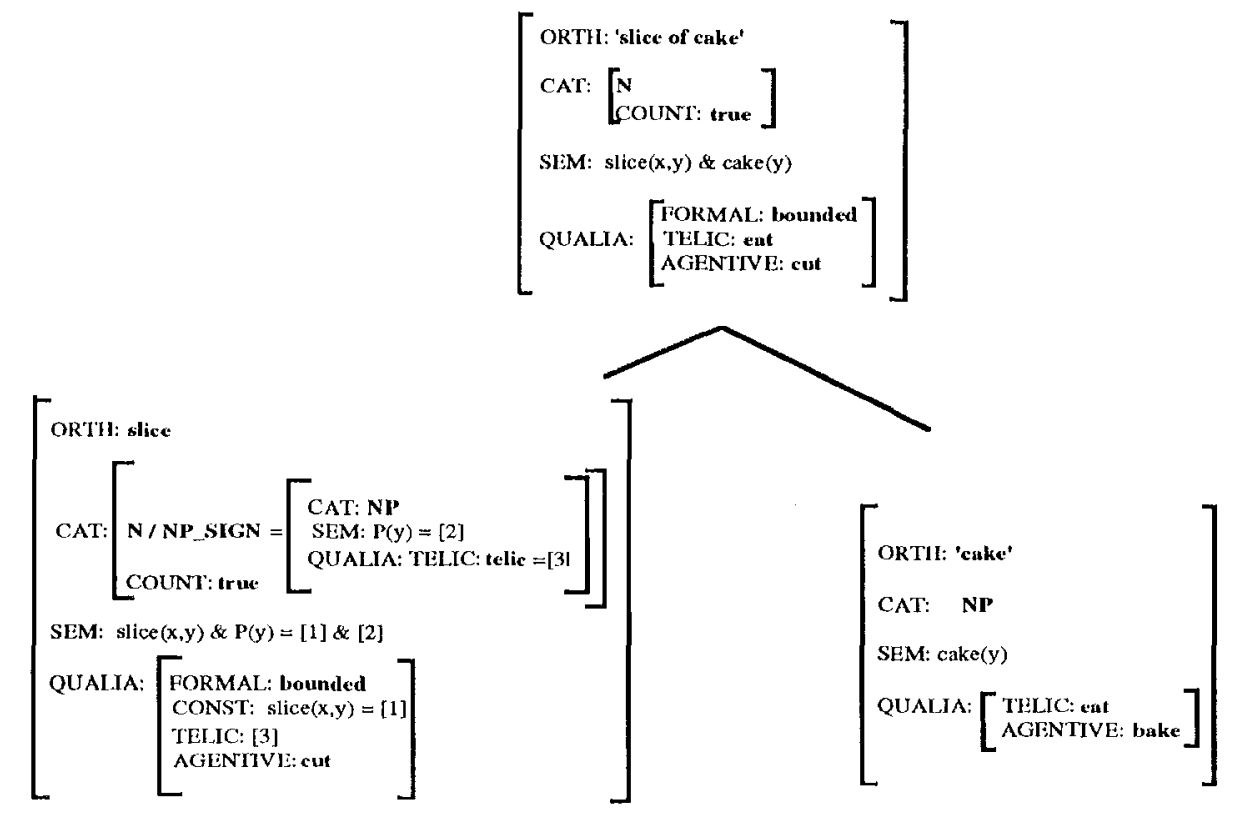

a tongue, box, cake, LIMON)

Figure 3. Composition

CONT: [-B] things (=substances, plurals/aggregates) (a 'container' of wheat, sugar, water, paper, cakes, tongues, boxes)

DTCHD: $[+B]$ entities (=individuated) (a 'detached portion' of a lemon, cake)

MDI.D: $[-\mathrm{B}]$ entities (=substances) ( $a$ 'shaped mass' of sugar, wheat, paper)

With respect to shape, it has to be noticed that while that of EL'T and MDLD is inherent to the portion itself (in SILT because the portion pre-existed as an individual; in MDLD because the whole was an amorphous mass and it is the process of portioning what has bounded the new thing), in BOUND and DTCHD shape is somehow relative to the whole. This way, a RODAJA is round because it is a cross-cut of either approximately-spherical (lemon) or cylindrical (sausage) objects; a slice of bread will be elliptic or square depending on whether the 'bread' is the classical loaf or the modern polyhedric-shaped one; top of a box will show identical behaviour.

Something similar happens with relative quantification. While the measure conveyed by CONT, EL'T and MIDLD is absolute, that of BOUND and DTCHD is relative: a top of a box or a slice of bread will be bigger or smaller depending on the magnitude of the box or the loaf of bread.

\section{Representation}

To represent PNs in the $\mathrm{LKB}$ we have made some interpretations for FORMAL and CONST Quales of the QUALIA.

We assume that the minimal and most basic FORMAL distinction among entities (as conceptualised by speakers) is that of their boundedness or not in terms of [IAC91]. Therefore, this Quale will be assigned to one of both types (or a coherent subtype). Similarly, the minimal constitutive distinction to be done is assumed to be that of entailment or not about internal structure of

things. Therefore, the CONST role will be assigned to one of both $[ \pm I]$ types (i_str_true/i_str_false). In this way, selectional restrictions of PNs will be basically defined as selection of signs bearing appropriate types for their FORMAL and CONST Quales.

As defined in [PUS95], SHAPE and magnitude (MAGN) are features of the FORMAL Role. Their values can be, as discussed above, either absolute or relative, depending on the kind of portion. In the latter cases, SHAPE and MAGN of portions will be a function of the corresponding values of the QUALIA of the whole ${ }^{2}$. This interpretation of the MAGN feature accounts for the nature of relative quantifiers of PNs.

At their turn, $[+\mathrm{I}]$ CONST's bear the feature ELTS c.g. rice:QUALIA:CONST:ELTS:grain. (vid. [JAC91], also [COP92]).

[PUS95] (assuming [VIK94] interpretation) attributes the CONST Role the function of representing the predicative relation Part-Of. Coherently, we assume CONST as encoding a relational predicate $R(x, y)$, being $R$ a type taxonomically daughter of Part-Of. In the default case, $\mathrm{R}$ will be 'Portion' and in more finc-grained cases, a daughter type of it -e.g. 'Slice'.

The Logical Semantics of PNs (SEM) will account for their both partitive and relational nature by adopting as predicate-argument structure that of their CONST Role. For the sake of unification, in the LKB, SEM will be the conjunction of this predicate and the SEM value of the sign denoting the whole. This way (vid.fig.3), that of 'slice of cake' will result in SLICE( $(x, y)$ \& CAKE(y) -thus accounting for the logics of John eats a slice of cake as EAT(e,John, $\mathrm{x}$ ) \& SI.ICE $(\mathrm{x}, \mathrm{y})$ \& CAKE(y) versus that of John eats cake as $\mathrm{EAT}(\mathrm{e}, \mathrm{Iohm}, \mathrm{x}) \& \mathrm{CAKE}(\mathrm{x})$.

2 [IAC91] develops a method to formalise relative shapes including judgements about dimensionality. 
For case of exposition, the syntax (CAT) of PNs is represented here as the complex category N/NP_sign, where the NI'sign is appropriately

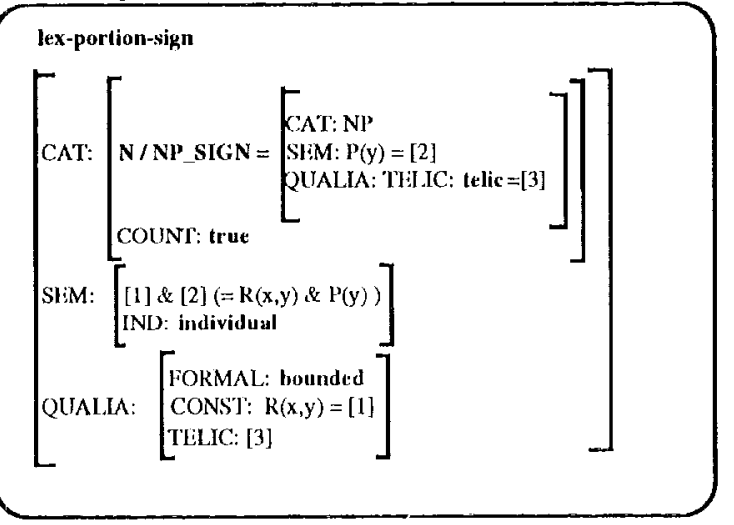

Figure 4. General Portion Sign

specilied to account for selectional restrictions and transitivity of properlies between the whole and the portion via feature reentrancics. This way, the composition of slice (N/NP) and cake (NI) will result in an $\mathrm{N}$ (slice of cake). This accounts for the fact that partitive constructions (c.g. slice of cake) do bear the combinatorial possibilities of nomns, while those of PNs are distinct and specific. The preposition (of) is omitted here -not in the I.KB implementation- since it is unrelevant as it lacks semantic content. We assume so [CHO81] analysis which considers of in this kind of constructions a mere surface case-marker. This view is conlimed by data of Spanish (UN VASO VINO) $=$ UN VASO DIE VINO, UN TROZOO PAN = UN 'IROKODL PAN) ['a glass wine' = 'a glass of wine', 'a portion bread' = 'a portion of bread'] in which the preposition is elidible -something which is not possible in the case of contentful prepositions (VLiNGO D) I: BARCIULONA $>$ *VINGO BARCLLONA) ['I-come from Batcelona' $\rightarrow$ *'I-come Barcelona'].

Moreover, CAT bears the feature COUN'l standing, as well for case of exposition, for the range of (surface) grammatical bchaviour of lexical signs usually referred to as countability/uncountability (sce discussion above).

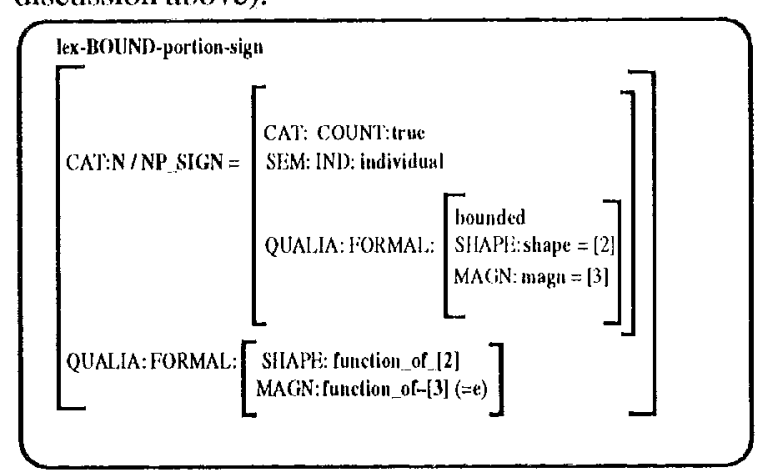

Figure 5. Boundary Portions

Provided all which has been discussed up to here, the general lex-PORTION-sign is defined as in fig.4; that is, as selecting NP's and resulting in FORMAL:[+B] entity-denoting signs (therefore individuated and syntactically countable) where the only QUALIA feature which percolate from the whole is the 'TELIC Role -the rest of Quales may be overridden by that of the PN.

Specific sub-types are shown in figs. 5 to 9. BOUNI) PNs (fig.5) select [+B] entities (therefore individuated and countable) (the tip of the tongue). Being $[+B]$, such wholes bear definite shape and magnitude; therefore such values for the portion will be functions of those of the whole. MAGN, specifically, is assumed to be conceptualised as somehow minimal, therefore notated 'e' ([JAC91]).

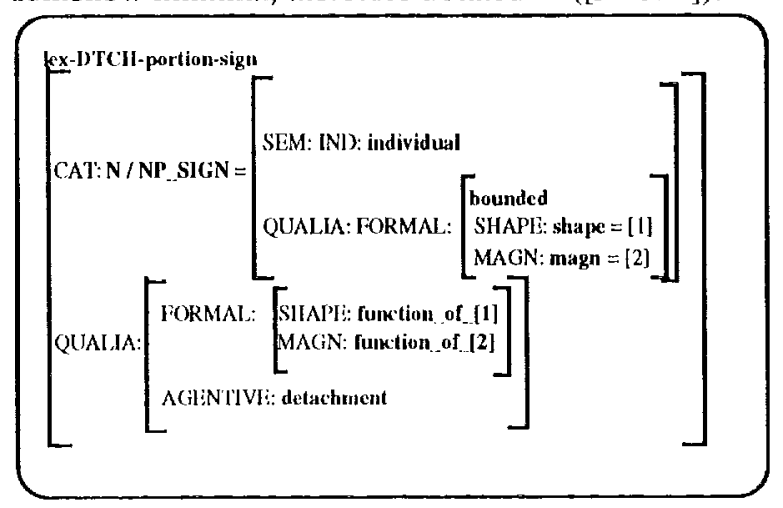

Figure 6, Detached lortions

Similarly, SHIAPE and MAGN of DTCIID PNs (fig.6) are functions of SHAPli and MAGN of the wholes they select. The difference with BOUND PNs is that the MAGN value doesn't tend to be minimal. 'The AGEN'IIVE: Role is here relevant -as it as well is in MJ)I.J) PNs.

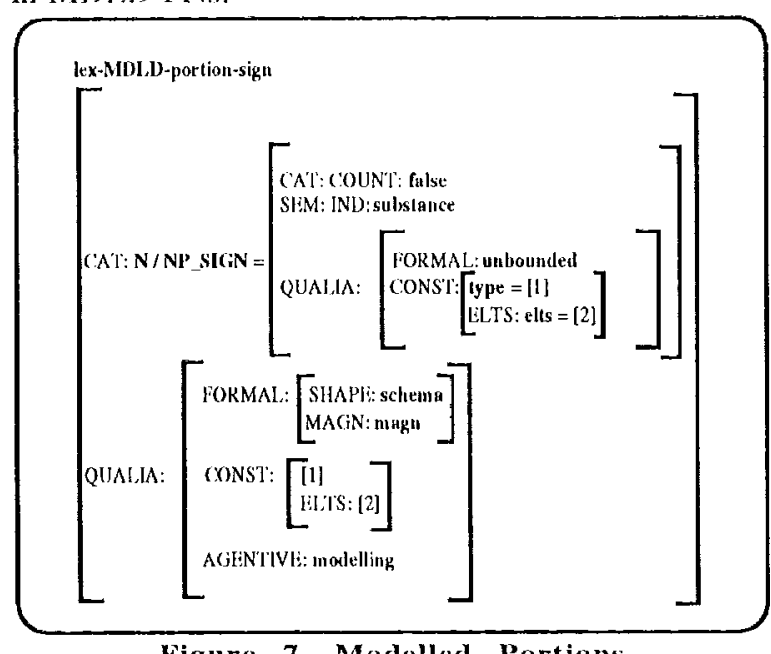

Figure 7. Modelled Portions

What is more remarkable in MDI,D PNs (fig.7) it that the CONST type of the whole (therefore its value for CONST:LLTS) is inherited by the portion -c.g., if 'sugar 'is $[+1]$ and consists of grains, a lump of sugar so; if 'paper 'has no entailment about internal structure, a sheet of paper has not either. M $\triangle$ GN is absolute (i.e., not related to that of the whole, since that is [-B]; e.g., in broad ontline, 'all the sugar/paper of the world'). SHAPl: is assumed to be schematic (vid. \$4).

SIIAPE and MAGN of LLT portions (fig.8) are also non-relative. These PNs select $[+I]$ entities, usually substances ('wheat', 'rice') but also possibly individuals ('lemon', as conventionalised in Spanish as internally-structured in GAIOS). The value for CONST:EL,TS of the whole will be the CONST 
predicate of the portion, thus its SEM predicate -e.g. rice:QUALIA:CONST:ELTS:grain $\Rightarrow$ grain:QUALIA:CONST:grain $(x, y) \Rightarrow$ grain-of-rice:SEM:grain( $x, y)$ \& $\operatorname{rice}(y)$.

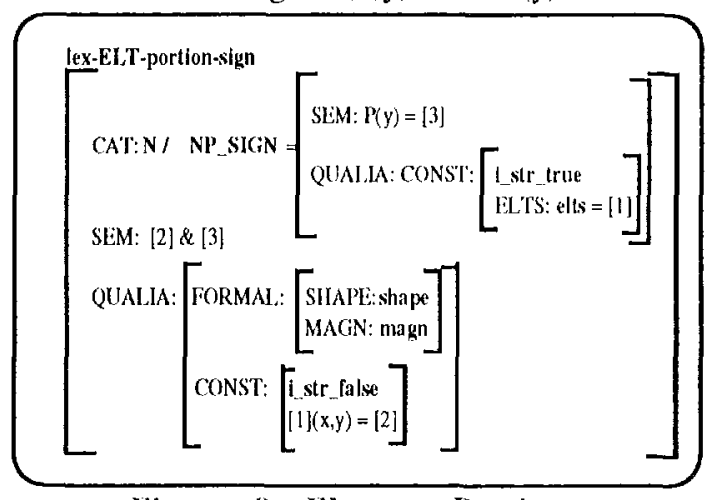

Figure 8. Element Portions

Last, CONT PNs (fig.9) select [-B] items (therefore substances but also plurals) ${ }^{3}$. The portion retains the constitution of the wholc. $\Lambda$ s discussed above, SIIAPE is not relevant, measure (MAGN) is. AGEN'TIVE, if considered relevant, will be a process of filling the container.

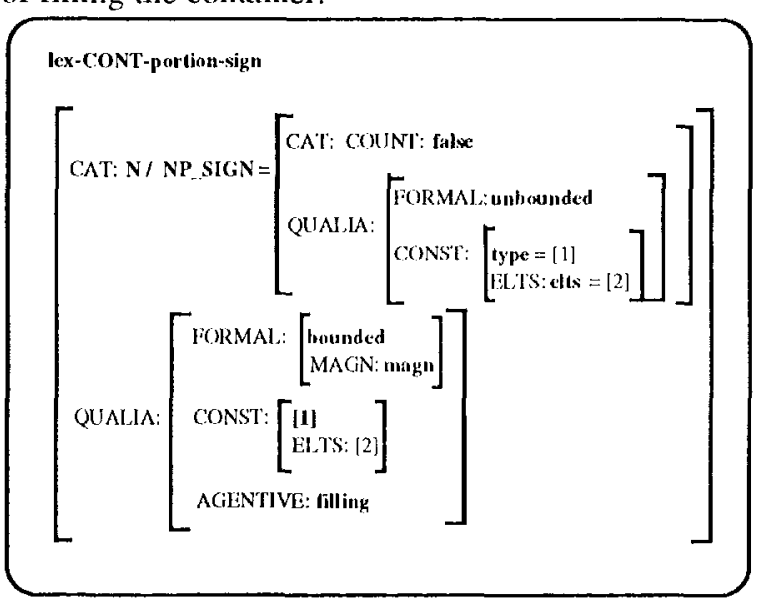

Figure 9, Container Portions

\section{Concluding Remarks}

In this paper we presented a system of representation of relational nouns denoting portions, segments and relative quantitics of entities according to the 'Theory of Qualia ([PUS95]), which has been parlially reinterpreted and adapted to the insights of Conceptual Semantics ([IAC91]). This system accounts for a range of linguistic facts, being the most remarkable the following:

1. Portions are mainly encoded in the language by means of constructions instead of by single lexical units

2. Portions are both bounded entities and relative measures of the wholes of reference

3. Portions inherit from their wholes their purpose or function, but, on the contrary, they show distinctive shape, magnitude and origin

3 Possibly also [-B] collections (groups) (a wagon of cattle), but, as said hefore, we're not commited here to discuss individuation of collections.
4. Partitive Nouns (PNs) select whole-denoting nouns according to the properties of the referent wholes as conceptualised by speakers

PNs have been classificd according to the semantic regularities they show, resulting in a system of five basic types, which have been represented in a unification formalism, LKB-LRL ([COP92]), allowing for composition of PNs (e.g. slice) and NPs (e.g. cake) (plus the scmantically void preposition 'of'; in Spanish, DE) into portion-denoting signs (e.g. slice of cake).

Acknowledgements. I'd like to thank Drs. 'T'oni Martí and Iloracio Rodriguez for their comments and support. This research is funded by the project PB-940830 of the DGICYT:

\section{References}

[CAR92] CARPENTER B. 1992. The Iogic of Typed Feature Structures. Cambridge University l'ress.

[CIA88] CIIAFFIN R. and IIERRMAN D.I. 1988. The nature of semantic relations. In Relational Models of the Lexicon, Martha Walton Evens ed. Cambridge U. Press [CHO81] CHOMSKY N. 1981. Lectures on Government and Binding. Foris, Dordrecht.

[COP92] COPLSTAKE, $\Lambda$. 1992. The Representation of Lexical Semantic Information. Ph. D. Thesis. University of Sussex (Cognitive Science research paper CSRP 280) [IRI88] IRIS M.A., LITOWTIZ B.E. and WALTON EVENS M. 1988. Problems of the part-whole relation. In Relational Models of the Lexicon, Martha Walton Evens ed. Cambridge University Press.

[IAC91] JACKISNDOFF R. 1991. Parts and Boundaries. In Lexical and Conceptual Semantics, Levin \& Pinker eds. Elsevier Science Publishers. Amsterdam.

[KRI87] KRIFKA, M. 1987. Nominal Reference and T'emporal Constitution: Towards a Semantics of Quantity. Proceedings of the 6th. Amsterdam Colloquium pp. 153-173. University of $\Lambda$ msterdam.

[LAN91] I.ANGACKER R. 1991. Foundations of Cognitive Grammar. Vol II. Deseriptive Application. Stanford University Press. Stanford, CA.

[LAK87] I AKOFF G. 1987. Women, Fire and Dangerous Things: What Categories Reveal about the Mind. University of Chicago Press. Chicago.

[LYO77] LYONS J. 1977. Semantics. Cambridge University Press. Cambridge.

[PUS95] PUSTEIOVSKY J. 1995. The generative I.exicon. The MIT Press. Cambridge, Ma.

[TAI.78] TAIMY, L. 1978. The Relation of Grammar to Cognition: A Synopsis. In D. Waltz (Ed.), Theoretical Issues in Natural Language Processing 2. New York: Association for Computing Machinery.

[VIK94] VIKNER C. and P. IIANSEN. 1994. Knowledge Bases and Generative Lexicons. Proc. of 'Workshop on Lexical Semantics', University of Copenhagen.

[VOS94] VOSSEN P. \& COPESTAKE A. 1994. Untangling Definition Structure into Knowledge Representation. In Briscoe, de Paiva, Copestake Eds. "Inheritance, Defaults, and the Iexicon", CUP, Cambridge.

[WAL88] WALTON EVLENS M. Ed. 1988. Relational Models of the Lexicon. Cambridge University Press.

[WIN87] WINSION M., CIIAFFIN R. and HERRMAN D. 1987. A Taxonomy of Part-Whole Relations. Cognitive Science $11,417-444$ 